\title{
Raining Frogs, Flying Horses, and Defining Tumor Rupture in GIST
}

\author{
E. A. Asare, MD, MS ${ }^{1}$, and B. W. Feig, $\mathrm{MD}^{\mathbf{1}}$ \\ Department of Surgical Oncology, University of Texas, M. D. Anderson Cancer Center, Houston, TX
}

Harmonizing definitions in surgical oncology is critical for several reasons which include: (1) the ability to pool data across institutions for robust statistical analysis in order to form decisions regarding patient care, (2) creation of a standard benchmark to allow comparisons of various published studies, and (3) provision of a common universal language for effective communication among surgeons. With regards to gastrointestinal stromal tumors (GIST), "tumor rupture" has not been consistently defined in many published studies over the years. ${ }^{1,2}$ While most studies have found an increased risk of recurrence and decreased survival for patients with "tumor rupture," others have not found any deleterious effect, likely due to the subjective addition of a variety of clinical scenarios that were included under the classification of "tumor rupture." 1-3

In this issue of Annals of Surgical Oncology, Nishida et al. ${ }^{4}$ propose definitions of "tumor rupture" based on minor modifications to the categories previously outlined by the Oslo sarcoma group and other published retrospective studies. ${ }^{1,3,5-7}$ They propose the following categories of "rupture" with regards to GIST: (1) tumor fracture or spillage, (2) blood-stained ascites, (3) gastrointestinal perforation at tumor site, (4) microscopic infiltration of an adjacent organ, (5) intralesional dissection or piecemeal resection, and (6) incisional biopsy. The efforts by Nishida et al. to harmonize the definition of GIST "rupture" are laudable, however, a call for adoption of the six proposed categories is premature, as there is no robust statistical or scientific basis of support provided by the authors. The level of evidence in support of this

\footnotetext{
(C) Society of Surgical Oncology 2019
}

First Received: 11 February 2019;

Published Online: 22 March 2019

B. W. Feig, MD

e-mail: bwfeig@mdanderson.org proposed definition is based on several retrospective reviews, which are all handicapped by a severely limited sample size. Despite the authors' assertion in the manuscript that their proposal for the definition of tumor rupture is "evidence based," the evidence presented to support their hypothesis is weak, at best. The evidence provided by the authors to support their proposed definition of tumor rupture comes mainly from three sources: the Oslo criteria for tumor rupture in small bowel GIST, the Oslo criteria for rupture in gastric GIST, and the Kinki GIST registry. To provide a standard definition of "tumor rupture" for GIST, the Oslo sarcoma group in 2016 proposed the following five scenarios as categories for the definition of tumor rupture: (1) spillage, fracture, or piecemeal removal, (2) bowel perforation at tumor site, (3) blood-tinged ascites, (4) microscopic infiltration of adjacent organs, and (5) surgical biopsy. ${ }^{3}$ This proposal was based on a retrospective review of 19 eligible patients with small bowel GIST treated in Norway from 2000 to 2012. By virtue of the very small sample size, the number of patients in each of the proposed "tumor rupture" categories were as follows: spillage, fracture, or piecemeal removal (eight), bowel perforation at tumor site (six), blood-tinged ascites (two), microscopic infiltration of adjacent organs (two), and surgical biopsy (one). The Oslo sarcoma group subsequently reported on recurrence-free survival outcomes for 22 patients with gastric GIST classified according to their proposed definition. ${ }^{5}$ It is worth mentioning that, in this validation study, there were no patients with "perforation" or "incisional biopsy," thus those two categories cannot be said to have been validated. The Kinki GIST registry group from Japan retrospectively reviewed their cases of "ruptured" GIST (study cohort 21, validation cohort 5) and found the median overall survival of patients with "tumor rupture" to be 6.4 years compared with 11.9 years without "rupture," although this could not be validated. ${ }^{1}$ Since this was a retrospective study, the definition of "rupture" was 
determined by the surgeon at time of surgery, and was therefore subjective and inherently inconsistent in its definition-exactly the problem that the authors of the current manuscript are attempting to address. It is counterintuitive to support a hypothesis with data which were collected by the same flawed means that is being critiqued. Additionally, with only five total patients in the validation portion of the study, it is impossible to obtain any statistical significance from this analysis. In order to ascertain whether the operative surgeons for the cohort concurred with the definitions proposed by the Oslo sarcoma group, a questionnaire was sent to 33 institutions in Japan with a reported survey response rate of $76 \%$. Recall bias, small sample size, and nonrepresentativeness of the surveyed population are important limitations of the study by the Kinki GIST registry group.

Another significant point of controversy lies with the inclusion of "microscopic infiltration of an adjacent organ" as a category included within the definition of tumor rupture. This requires a major leap of faith for several reasons. Traditionally, tumor rupture in the "common sense" scenario is a clinical and not a pathological finding. Lumping "microscopic infiltration of adjacent organs" together with "tumor fracture or spillage" based on the observation that patients with both processes have similar outcomes requires a complete redefinition of long-established concepts of tumor biology. Although the argument offered by the authors to justify this change may in time be shown to be a pathophysiologic possibility, we should not change well-established scientific, biologic processes based on a "theory"- a change of this magnitude to scientific dogma requires data, something again that is severely lacking in this manuscript. It is not scientifically or statistically valid to assume that, because two clinical endpoints are similar, they are the result of the same biologic process. More than likely, this represents a true, true, but unrelated scenario. Simply put, just because you see frogs after it rains, does not mean that it rained frogs. Changes in established principles of tumor biology could have unanticipated implications for patient care, including but not limited to the role of adjuvant therapies, surveillance, and patient anxiety. In a similar vein, we agree with the authors that image-guided biopsy is encouraged and the preferred method for obtaining tissue for pathologic evaluation, but there are rare instances where an incisional biopsy is warranted. To classify deliberate, controlled surgical biopsy as "rupture" in the absence of convincing data could potentially be deleterious to patient care.

The biological, molecular, and anatomic profile of mesenchymal tumors differs from epithelial-derived tumors, thus separate definitions of "tumor rupture" may be necessary. ${ }^{7}$ However, harmonizing definitions of "tumor rupture" on the histologic basis of different mesenchymal tumors may be impossible, as the rarity of these tumors will make it extremely difficult to obtain an adequate sample size needed to show statistical and clinical significance.

Establishing consistent, reproducible definitions of clinical phenomena is important for both patient care and clinical research. The definition of "tumor rupture" should be practical, unambiguous, and based on clinically reproducible data. In the article by Nishida et al., ${ }^{4}$ the authors propose descriptive clinical categories that they hypothesize should constitute the definition of tumor rupture in GIST. Acceptance of these new categories would require significant alteration in the traditionally accepted definition of tumor rupture. While this new definition of tumor rupture may, in time, be proven to be accurate, we feel strongly that such a dramatic change in the definition of tumor rupture should be based on reproducible scientific data. Unfortunately, these data are not provided in the manuscript due to the fact that the clinical phenomenon of tumor rupture in GIST is so rare that the data do not exist. What the authors have accomplished in this manuscript is to better elucidate prognostic factors in patients with GIST that are potentially predictive of clinical outcome. There are a number of ways that the authors could attempt to prove that the observations and hypotheses that they propose are scientifically valid, including but not limited to, eliciting the participation of multiinstitutional collaborative societies such as the American Joint Committee on Cancer (AJCC) or the Transatlantic Australasian Retroperitoneal Sarcoma Working Group (TARPSWG) to prospectively collect the clinical data necessary to verify their hypothesis. We are concerned that the random redefinition of classic, scientifically established clinical definitions without the appropriate scientific data to support those changes could potentially result in inappropriate changes in treatment algorithms which could have a negative impact on patient care and outcomes. We must be cautious when making significant changes such as those described, so that anecdotal similarities in behavior and outcome do not result in changes in long-standing, established clinical definitions based on erroneous assumptions. After all, "if you see a horse in the meadow, you can call the horse a bird; you can tell the horse it is a bird; however, despite changing the name, the horse will still not be able to fly." Until we have data to show that there is a scientific basis to support adding additional clinical scenarios (e.g., microscopic infiltration of adjacent organ) that were not classically included under the umbrella of tumor rupture, we should continue to use the definitions which are established: tumor rupture is the spillage or fracture of tumor into a body cavity. Only through prospective evaluation of patient outcomes, along with multidisciplinary and multiinstitutional discussion, will we be able to 
determine whether it is beneficial and scientifically valid to change long-standing, established definitions. Until then, a horse will remain a horse, and not be able to fly.

\section{REFERENCES}

1. Nishida T, Cho H, Hirota S, et al. Clinicopathological features and prognosis of primary GISTs with tumor rupture in the real world. Ann Surg Oncol. 2018;25:1961.

2. Rutkowski P, Bylina E, Wozniak A, et al. Validation of the Joensuu risk criteria for primary resectable gastrointestinal stromal tumour: the impact of tumour rupture on patient outcomes. Eur J Surg Oncol. 2011;37:890-6.

3. Holmebakk T, Bjerkehagen B, Boye $\mathrm{K}$, et al. Definition and clinical significance of tumour rupture in gastrointestinal stromal tumours of the small intestine. Br J Surg. 2016;103:684-691.
4. Nishida T, Hølmebakk T, Raut CP, Rutkowski P. Defining tumor rupture in gastrointestinal stromal tumor. Ann Surg Oncol. 2019. https://doi.org/10.1245/s10434-019-07297-9.

5. Holmebakk T, Hompland I, Bjerkehagen B, et al: Recurrence-free survival after resection of gastric gastrointestinal stromal tumors classified according to a strict definition of tumor rupture: a population-based study. Ann Surg Oncol. 2018;25:1133-9.

6. Enneking WF. A system of staging musculoskeletal neoplasms. Instr Course Lect. 1988;37:3-10.

7. Eriksen MT, Wibe A, Syse A, et al. Inadvertent perforation during rectal cancer resection in Norway. Br J Surg. 2004;91:210-6.

Publisher's Note Springer Nature remains neutral with regard to jurisdictional claims in published maps and institutional affiliations. 